\title{
Mass Effect on Axial Charge Dynamics
}

\author{
Er-dong Guo*1,3 and Shu $\operatorname{Lin}^{\dagger 2}$ \\ ${ }^{1}$ State Key Laboratory of Theoretical Physics, Institute of Theoretical Physics, \\ Chinese Academy of Sciences, Beijing 100190, China \\ ${ }^{2}$ Institute of Astronomy and Space Sciences, Sun Yat-Sen University, No 135 \\ Xingang Xi Rd, Guangzhou 510275, China \\ ${ }^{3}$ Kavli Institute of Theoretical Physics China, Chinese Academy of Sciences, Beijing \\ 100190, China
}

October 19, 2018

\begin{abstract}
We studied effect of finite quark mass on the dynamics of axial charge using the D3/D7 model in holography. The mass term in axial anomaly equation affects both the fluctuation (generation) and dissipation of axial charge. We studied the dependence of the effect on quark mass and external magnetic field. For axial charge generation, we calculated the mass diffusion rate, which characterizes the helicity flipping rate. The rate is a non-monotonous function of mass and can be significantly enhanced by the magnetic field. The diffusive behavior is also related to a divergent susceptibility of axial charge. For axial charge dissipation, we found that in the long time limit, the mass term dissipates all the charge effectively generated by parallel electric and magnetic fields. The result is consistent with a relaxation time approximation. The rate of dissipation through mass term is a monotonous increasing function of both quark mass and magnetic field.
\end{abstract}

*guoerdong@itp.ac.cn

${ }^{\dagger}$ linshu8@mail.sysu.edu.cn 


\section{Introduction}

It is believed that parity odd domains with chiral imbalance are produced in finite temperature quark-gluon plasma (QGP). Their presence can be detected via axial anomaly as chiral magnetic effect (CME) [1-4] and chiral magnetic wave (CMW) [5, 6] in heavy ion collisions, see [7 9] for recent reviews. The former leads to the generation of vector current along the direction of external magnetic field:

$$
\vec{j}_{V}=\frac{N_{c} e}{2 \pi^{2}} \mu_{5} \vec{B}
$$

where $\mu_{5}$ is the axial chemical potential characterizing the chiral imbalance. The latter leads to the propagation of axial and vector charges along the direction of external magnetic field. Analogous effects exist when the magnetic field is replaced by vorticity of QGP [10, 11]. These effects are being intensively searched for in heavy ion collision experiments in recent years 12 14].

Theoretical descriptions of CME and CMW have been developed in different frameworks including hydrodynamics [10,15, 18] and kinetic theory [19 25] etc. Most frameworks assume quarks being massless, see exception for example in [26,27]. While it is known that finite quark mass does not modify CME, we do expect quark mass to have imprints on the dynamics of axial charge. Naively, if the mass of one quark flavor is much larger than the temperature of QGP, that quark flavor decouples from axial current. We would like to ask quantitative questions on the mass effect on dynamics of axial charge. This is relevant in reality because the mass of strange quark is comparable to the temperature of QGP created at relativistic heavy ion collider (RHIC) and large hadron collider (LHC). With the inclusion of mass term, the axial anomaly equation reads

$$
\partial_{\mu} j_{5}^{\mu}=2 i m \bar{\psi} \gamma^{5} \psi-\frac{e^{2}}{16 \pi^{2}} \epsilon^{\mu \nu \rho \sigma} F_{\mu \nu} F_{\rho \sigma}-\frac{g^{2}}{16 \pi^{2}} \operatorname{tr} \epsilon^{\mu \nu \rho \sigma} G_{\mu \nu} G_{\rho \sigma},
$$

where the three terms on the right hand side (RHS) corresponds to mass term, QED anomaly term and QCD anomaly term respectively. (2) is written for one flavor of quark with mass $m$. All three terms lead to modification of axial charge dynamics. The effect of QED anomaly term is extensively studied in the above mentioned references. The effect of QCD anomaly was studied recently [28 31]. In this work, we will focus on the effect of the mass term. On one hand, finite quark mass explicitly breaks axial symmetry, offering a mechanism of axial charge generation. We find that the mass operator diffuses at low frequency the same way as the Chern-Simon (CS) number. The diffusion of the CS number is known to generate axial charge. The same is true for the mass operator. We calculate the diffusion rate of mass 
term as a measure of axial charge generation. We also define a dynamical susceptibility by $\mathrm{CME}$, and find it to be divergent in the low frequency limit. We explain the common physical reason for the diffusive mass operator and the divergent susceptibility. On the other hand, finite quark mass also leads to axial charge dissipation. The dissipation effect is studied recently in 32,33 in a relaxation time approximation. We will discuss axial charge dissipation in an indirect way: we set up parallel electric and magnetic field and measure the rate of dissipation through the mass term. The situation is further complicated by the existence of a reservoir of adjoint matter, to which axial charge can dissipate. By taking into account the additional loss rate, we find that the axial charge dissipates entirely in the long time limit, which is consistent with the relaxation time approximation. We will study these effects as a function of both quark mass and external magnetic field using a holographic model.

The paper is organized as follows: In Sec II we give a self-contained review of the holographic model. In Sec III we discuss separately mass effect on axial charge generation and dissipation, which we coined mass diffusion rate and mass dissipation effect respectively. We summarize the results in Sec IV. We collect technical details in obtaining phase diagram and hydrodynamic solutions in two appendices.

\section{A quick review of the model}

\subsection{The D3/D7 background}

We use the D3/D7 model to study the effect of finite quark mass. The background is sourced by $N_{c}$ D3 branes. The worldvolume fields of D3 branes are $\mathcal{N}=4$ supersymmetric YangMills (SYM) theory. In addition, there are $N_{f}$ D7 branes in the background. The open string stretching between D3 and D7 branes is dual to $\mathcal{N}=2$ hypermultiplet. The $\mathcal{N}=4$ and $\mathcal{N}=2$ fields are in the adjoint and fundamental representations of the $S U\left(N_{c}\right)$ group respectively. By analogy with $\mathrm{QCD}$, we will loosely refer to the $\mathcal{N}=4$ and $\mathcal{N}=2$ fields as gluons and quarks respectively. A detailed account of field content can be found in [34]. The $\mathcal{N}=4$ theory has a $S O(6)_{R}$ global symmetry, which is broken by the $\mathcal{N}=2$ theory to $S O(4) \times U(1)_{R}$. As we will see, the $U(1)_{R}$ symmetry is anomalous. We will identify it with axial symmetry. We start with the finite temperature black hole background of D3 branes 
following the notations of [35]:

$$
\begin{aligned}
d s^{2} & =g_{t t} d t^{2}+g_{x x} d \vec{x}^{2}+g_{\rho \rho} d \rho^{2}+g_{\theta \theta} d \theta^{2}+g_{\phi \phi} d \phi^{2}+g_{S S} d \Omega_{3}^{2}, \\
& =-\frac{r_{0}^{2}}{2} \frac{f^{2}}{H} \rho^{2} d t^{2}+\frac{r_{0}^{2}}{2} H \rho^{2} d x^{2}+\frac{d \rho^{2}}{\rho^{2}}+d \theta^{2}+\sin ^{2} \theta d \phi^{2}+\cos ^{2} \theta d \Omega_{3}^{2} .
\end{aligned}
$$

where

$$
f=1-\frac{1}{\rho^{4}}, \quad H=1+\frac{1}{\rho^{4}} .
$$

The temperature is fixed by $T=r_{0} / \pi$. Note that we have factorized $S_{5}$ into $S_{3}$ and two additional angular coordinates $\theta$ and $\phi$, which makes the breaking of global symmetry $S O(6)_{R} \rightarrow S O(4) \times U(1)_{R}$ manifest. There is a nontrivial background Ramond-Ramond form

$$
C_{4}=\left(\frac{r_{0}^{2}}{2} \rho^{2} H\right)^{2} d t \wedge d x_{1} \wedge d x_{2} \wedge d x_{3}-\cos ^{4} \theta d \phi \wedge d \Omega_{3} .
$$

In the probe limit $N_{f} / N_{c} \ll 1$, the D7 branes do not backreact on the background of the D3 branes. This corresponds to the quenched limit of QCD. The D3 and D7 branes occupy the following dimensions.

\begin{tabular}{l|cccccccccc} 
& $x_{0}$ & $x_{1}$ & $x_{2}$ & $x_{3}$ & $x_{4}$ & $x_{5}$ & $x_{6}$ & $x_{7}$ & $x_{8}$ & $x_{9}$ \\
\hline D3 & $\times$ & $\times$ & $\times$ & $\times$ & & & & & & \\
D7 & $\times$ & $\times$ & $\times$ & $\times$ & $\times$ & $\times$ & $\times$ & $\times$ & &
\end{tabular}

The D3 and D7 branes are separated in the $x_{8}-x_{9}$ plane. Using translation symmetry, we put D3 branes at the origin of the plane and parameterize the position of D7 branes by radius $\rho \cos \theta$ and polar angle $\phi$. The $\mathrm{D} 7$ branes have rotational symmetry in the $x_{8}-x_{9}$ plane, corresponding to $U(1)_{R}$ symmetry in the dual field theory. We use the symmetry to choose $\phi=0$. The embedding function $\theta(\rho)$ of D7 branes in D3 background is determined by minimizing the action including a DBI term and WZ term

$$
\begin{aligned}
& S_{D 7}=S_{D B I}+S_{W Z}, \\
& S_{D B I}=-N_{f} T_{D 7} \int d^{8} \xi \sqrt{-\operatorname{det}\left(g_{a b}+\left(2 \pi \alpha^{\prime}\right) \tilde{F}_{a b}\right)}, \\
& S_{W Z}=\frac{1}{2} N_{f} T_{D 7}\left(2 \pi \alpha^{\prime}\right)^{2} \int P\left[C_{4}\right] \wedge \tilde{F} \wedge \tilde{F} .
\end{aligned}
$$

Here $T_{D 7}$ is the D7 brane tension. $g_{a b}$ and $\tilde{F}_{a b}$ are the induced metric and worldvolume field strength respectively. Defining

$$
\begin{aligned}
& F_{a b}=\left(2 \pi \alpha^{\prime}\right) \tilde{F}_{a b}, \\
& \mathcal{N}=N_{f} T_{D 7} 2 \pi^{2}=\frac{N_{f} N_{c}}{(2 \pi)^{4}},
\end{aligned}
$$


we simplify the action to

$$
\begin{aligned}
& S_{D B I}=-\frac{\mathcal{N}}{2 \pi^{2}} \int d^{8} \xi \sqrt{-\operatorname{det}\left(g_{a b}+F_{a b}\right)}, \\
& S_{W Z}=\frac{1}{4 \pi^{2}} \mathcal{N} \int P\left[C_{4}\right] \wedge F \wedge F .
\end{aligned}
$$

The mass of the quark is realized as the separation of the D7 branes from the D3 branes at infinity. Explicitly, the mass $M$ is determined from the asymptotic behavior of $\theta$ :

$$
\sin \theta=\frac{m}{\rho}+\frac{c}{\rho^{3}}+\cdots
$$

with $M=r_{0} m$. We will turn on a constant magnetic field, which amounts to including worldvolume magnetic field in D7 branes. There are two possible embeddings with D7 branes crossing/not crossing the black hole horizon, corresponding to meson melting/mesonic phase respectively [35-38]. Using $t, \vec{x}, \rho$ and angular coordinates on $S_{3}$ as worldvolume coordinates, the induced metric is given by

$$
d s_{\text {ind }}^{2}=-\frac{r_{0}^{2}}{2} \frac{f^{2}}{H} \rho^{2} d t^{2}+\frac{r_{0}^{2}}{2} H \rho^{2} d \vec{x}^{2}+\left(\frac{1}{\rho^{2}}+\theta^{\prime}(\rho)^{2}\right) d \rho^{2}+\cos ^{2} \theta d \Omega_{3}^{2} .
$$

We also turn on a constant magnetic field in $z$-direction: $F_{x y}=B$, the action of D7 branes can be written as

$$
S_{D B I}=-\mathcal{N} \int d \rho\left(\frac{r_{0}^{2}}{2}\right)^{2} f H \rho^{3} \sqrt{1+\rho^{2} \theta^{\prime 2}} \sqrt{1+\frac{2 B^{2}}{r_{0}^{2} H \rho^{2}}} \cos ^{3} \theta,
$$

with a vanishing WZ term. The phase diagram in the $m$ - $B$ plane has been obtained in [37, 38]. We reproduce the result in appendix A and show the result at fixed temperature in Figure. 1. The two phases are mesonic phase with larger $m$ and $B$ and meson melting phase with smaller $m$ and $B$. In the former case, R-charge (axial charge) exchange between fundamental matter and adjoint sector is not possible due to the formation of meson bound state, while in the latter case, R-charge (axial charge) can leak from fundamental matter to adjoint sector. The phase diagram implies that large quark mass and magnetic field favors formation of meson bound state. The effect of magnetic field may be understood via an increased effective quark mass. We are interested in the meson melting phase, which is more relevant for application in QGP. 


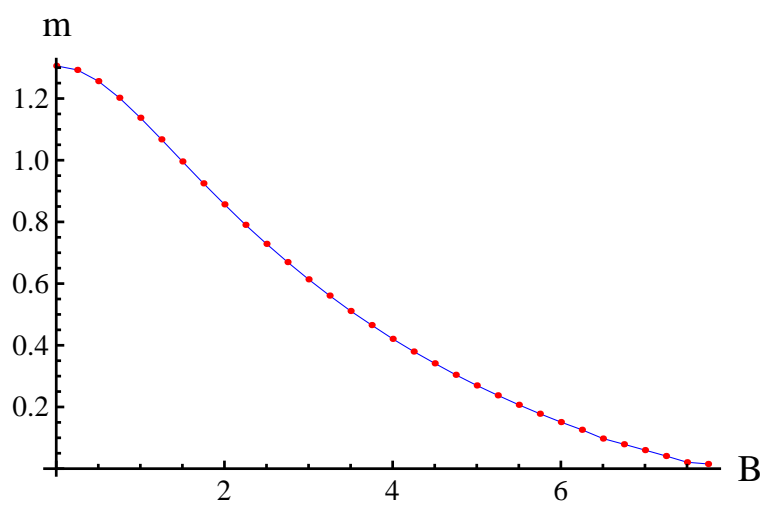

Figure 1: $m-B$ phase diagram of $D 3 / D 7$ background. The axis labels are dimensionless numbers with units set by $\pi T=1$. The region with small $m$ and $B$ corresponds to the meson melting phase, while the region with large $m$ and $B$ corresponds to the mesonic phase.

\subsection{Fluctuations and realization of axial anomaly}

We consider the fluctuation of embedding function $\phi$ and worldvolume gauge field $A_{M}$. The quadratic action can be written in the following compact form

$S=\mathcal{N} \int d^{5} x\left(-\frac{1}{2} \sqrt{-G} G^{M N} \partial_{M} \phi \partial_{N} \phi-\frac{1}{4} \sqrt{-H} F^{2}\right)-\mathcal{N} \kappa \int d^{5} x \Omega \epsilon^{M N P Q R} F_{M N} F_{P Q} \partial_{R} \phi$,

where $M=t, x_{1}, x_{2}, x_{3}, \rho$. The EOM of $\phi$ is given by

$$
\frac{\delta S}{\delta \phi}-\partial_{M}\left(\frac{\delta S}{\delta \partial_{M} \phi}\right)=0 .
$$

Since $\phi$ is a phase, only its derivative enters the action, we have from (14),

$$
\partial_{\mu}\left(\frac{\delta S}{\delta \partial_{\mu} \phi}\right)+\partial_{\rho}\left(\frac{\delta S}{\delta \partial_{\rho} \phi}\right)=0
$$

with $\mu=t, x_{1}, x_{2}, x_{3}$. Defining $J_{R}^{\mu}=\int d \rho \frac{\delta S}{\delta \partial_{\mu} \phi}$, we obtain

$$
\partial_{\mu} J_{R}^{\mu}+\left.\frac{\delta S}{\delta \partial_{\rho} \phi}\right|_{\rho=\rho_{h}} ^{\infty}=0
$$

We will identify $J_{R}$ as the axial current. The non-conservation of $J_{R}$ follows from two boundary terms in the integration. The boundary term at the horizon $\rho=\rho_{h}$ indicates axial charge exchange between D7 branes and D3 branes. It is pointed out in [34 that this term represents leakage of $\mathrm{R}$-charge from fundamental sector to adjoint sector as fields in 
both sectors are charged under the $U(1)_{R}$ symmetry. The other boundary term at $\rho=\infty$ can be related to axial anomaly:

$$
O_{\phi} \equiv-\left.\frac{\delta S}{\delta \partial_{\rho} \phi}\right|_{\rho=\infty}=-\frac{\delta S^{\partial}}{\delta \phi(\rho \rightarrow \infty)}
$$

where we have used the defining property of on-shell action $S^{\partial}$. For action (13), we have

$$
O_{\phi}=\left.\mathcal{N} \sqrt{-G} G^{M \rho} \partial_{M} \phi\right|_{\rho=\infty}+\left.\kappa \mathcal{N} \Omega \epsilon^{M N P Q} F_{M N} F_{P Q}\right|_{\rho=\infty}
$$

For our model,

$$
\begin{aligned}
& \sqrt{-G} G^{M N}=\sqrt{-h} g^{M N} g_{\phi \phi}, \quad \sqrt{-H}=\sqrt{-h}, \\
& \Omega=\cos ^{4} \theta, \quad \kappa=\frac{1}{8}
\end{aligned}
$$

with $h$ to be defined in the next section. Field theory analysis shows that 34$]$

$$
O_{\phi}=m i \bar{\psi} \gamma^{5} \psi+\cdots+\mathcal{N} E \cdot B
$$

where $\cdots$ represents contribution from supersymmetric partners. Noting that $\theta \rightarrow 0$ as $\rho \rightarrow \infty$, we readily identify the second term in (18) with the last term in (20). The remaining term in (18) can then be identified with the mass term in (20). For convenience, we define the remaining term by

$$
O_{\eta}=\left.\mathcal{N} \sqrt{-G} G^{M \rho} \partial_{M} \phi\right|_{\rho=\infty}
$$

We have thus holographically split $O_{\phi}$ into the mass term $O_{\eta}$ and anomaly term $\mathcal{N} E \cdot B$, which represent respectively explicit and anomalous breaking of axial symmetry:

$$
\partial_{\mu} J_{R}^{\mu}=O_{\phi}=O_{\eta}+\mathcal{N} E \cdot B
$$

\section{Finite Quark Mass Effect}

We will study two aspects of finite quark mass effect: i, the mass term, similar to QCD anomaly term, has diffusive behavior at low frequency. This gives rise to fluctuation (random walk behavior) of axial charge. The rate of diffusion, to be referred to as mass diffusion rate, determines the rate of axial charge generation; ii, in the presence of nonvanishing $E \cdot B$, net axial charge would be produced. However, the axial charge dissipates due to finite quark mass, resulting in a reduced rate of axial charge generation. We will refer to

\footnotetext{
${ }^{1}$ Note that we have $\phi=0$, thus no axial chemical potential is introduced.
} 
this as mass dissipation effect. The above effects are captured by correlators of $J^{z}$ and $O_{\eta}$. The mass diffusion rate involves the correlator of $O_{\eta}$ itself, while the mass dissipation effect involves the correlator between $J^{z}$ and $O_{\eta}$. We stress that $J^{z}$ is the vector current coupled to boundary gauge field $A_{z}$. In holographic formulation, we need to study the fluctuation of bulk fields $A_{z}$ and $\phi$, which are dual to $J^{z}$ and $O_{\eta}\left(O_{\phi}\right)$. For our purpose, it is sufficient to turn on homogeneous (in both $\vec{x}$ and $S_{3}$ ) fluctuation of $A_{z}(t, \rho)$ and $\phi(t, \rho)$. The fluctuation leads to the following modification of the following quantities

$$
\begin{aligned}
& d s_{\text {ind }}^{2}=\left(g_{t t}+g_{\phi \phi} \dot{\phi}^{2}\right) d t^{2}+g_{x x} d x^{2}+\left(g_{\rho \rho}+g_{\theta \theta} \theta^{\prime 2}+g_{\phi \phi} \phi^{\prime 2}\right) d \rho^{2}+g_{S S}^{2} d \Omega_{3}^{2}+2 g_{\phi \phi} \dot{\phi} \phi^{\prime} d t d \rho, \\
& \delta F=\dot{A}_{z} d t \wedge d z+A_{z}^{\prime} d \rho \wedge d z \\
& \delta P\left[C_{4}\right]=-\cos ^{4} \theta\left(\dot{\phi} d t+\phi^{\prime} d \rho\right) \wedge d \Omega_{3} .
\end{aligned}
$$

With (23), we can write down the quadratic action of $A_{z}(t, \rho)$ and $\phi(t, \rho)$ :

$$
\begin{aligned}
S_{\mathrm{DBI}}+S_{\mathrm{WZ}} & =-\mathcal{N} \int d t d^{3} x d \rho\left[\frac{1}{2} \sqrt{-h}\left(g^{t t} g_{\phi \phi} \dot{\phi}^{2}+g^{\rho \rho} g_{\phi \phi} \phi^{2}+g^{t t} g^{x x} \dot{A}_{z}^{2}+g^{\rho \rho} g^{x x} A_{z}^{\prime 2}\right)\right. \\
& \left.+\cos ^{4} \theta B\left(\phi^{\prime} \dot{A}_{z}-\dot{\phi} A_{z}^{\prime}\right)\right]
\end{aligned}
$$

where we have defined

$$
\sqrt{-h}=\sqrt{-g_{t t} g_{x x}\left(g_{x x}^{2}+B^{2}\right)\left(1+\rho^{2} \theta^{\prime 2}\right) g_{\rho \rho} g_{S S}^{3}} .
$$

Variation with respect to the fluctuations gives both the EOM and the on-shell action

$$
\begin{aligned}
\delta S & =-\mathcal{N} \int d t d^{3} x d \rho[\sqrt{-h} \times \\
& \left(-\partial_{t}\left(g^{t t} g_{\phi \phi} \dot{\phi}\right) \delta \phi-\partial_{\rho}\left(g^{\rho \rho} g_{\phi \phi} \phi^{\prime}\right) \delta \phi-\partial_{t}\left(g^{t t} g^{x x} \dot{A}_{z}\right) \delta A_{z}-\partial_{\rho}\left(g^{\rho \rho} g^{x x} A_{z}^{\prime}\right) \delta A_{z}\right) \\
& \left.-\partial_{\rho}\left(\cos ^{4} \theta B \dot{A}_{z}\right) \delta \phi+\partial_{t}\left(\cos ^{4} \theta B A_{z}^{\prime}\right) \delta \phi-\partial_{t}\left(\cos ^{4} \theta B \phi^{\prime}\right) \delta A_{z}+\rho\left(\cos ^{4} \theta B \dot{\phi}\right) \delta A_{z}\right] \\
& -\mathcal{N} \int d t d^{3} x\left[\sqrt{-h}\left(g^{\rho \rho} g_{\phi \phi} \phi^{\prime} \delta \phi+g^{\rho \rho} g^{x x} A_{z}^{\prime} \delta A_{z}\right)+\cos ^{4} \theta B\left(\dot{A}_{z} \delta \phi-\dot{\phi} \delta A_{z}\right)\right] .
\end{aligned}
$$

Working with a single Fourier mode $e^{-i \omega t}$, we obtain the EOM

$$
\begin{aligned}
& \omega^{2} \sqrt{-h} g^{t t} g_{\phi \phi} \phi-\partial_{\rho}\left(\sqrt{-h} g^{\rho \rho} g_{\phi \phi} \phi^{\prime}\right)-B \partial_{\rho}\left(\cos ^{4} \theta\right) A_{z}(-i \omega)=0 \\
& \omega^{2} \sqrt{-h} g^{t t} g^{x x} A_{z}-\partial_{\rho}\left(\sqrt{-h} g^{\rho \rho} g^{x x} A_{z}^{\prime}\right)+B \partial_{\rho}\left(\cos ^{4} \theta\right) \phi(-i \omega)=0 .
\end{aligned}
$$

The asymptotic expansion of $\phi$ and $A_{z}$ can be determined from EOM:

$$
\begin{aligned}
& \phi=f_{0}+\frac{f_{1}}{\rho^{2}}+\frac{f_{h}}{\rho^{2}} \ln \rho+\cdots, \\
& A_{z}=a_{0}+\frac{a_{1}}{\rho^{2}}+\frac{a_{h}}{\rho^{2}} \ln \rho+\cdots
\end{aligned}
$$


$f_{0}$ and $a_{0}$ correspond to sources coupled to $O_{\phi}$ and $J^{z}$. The coefficients of the logarithmic terms correspond to counter term:2:

$$
f_{h}=\frac{\omega^{2}}{r_{0}^{2}} f_{0}, \quad a_{h}=\frac{\omega^{2}}{r_{0}^{2}} a_{0}
$$

The vevs of $O_{\phi}$ and $J^{z}$ are determined by

$$
\begin{aligned}
O_{\phi} & =\frac{\delta S^{\partial}}{\delta \phi(\rho \rightarrow \infty)} \\
& =\left.\left(-\mathcal{N} \sqrt{-h} g^{\rho \rho} g_{\phi \phi} \phi^{\prime}-\mathcal{N} \cos ^{4} \theta B \dot{A}_{z}\right)\right|_{\rho \rightarrow \infty}=2 \mathcal{N}\left(\frac{r_{0}^{2}}{2}\right)^{2} m^{2} f_{1}-\mathcal{N} B a_{0}(-i \omega), \\
J^{z} & =\frac{\delta S^{\partial}}{\delta A_{z}(\rho \rightarrow \infty)} \\
& =\left.\left(-\mathcal{N} \sqrt{-h} g^{\rho \rho} g^{x x} A_{z}^{\prime}+\mathcal{N} \cos ^{4} \theta B \dot{\phi}\right)\right|_{\rho \rightarrow \infty}=2 \mathcal{N}\left(\frac{r_{0}^{2}}{2}\right) a_{1}+\mathcal{N} B f_{0}(-i \omega),
\end{aligned}
$$

where we have used $\left.\rho \sin \theta\right|_{\rho \rightarrow \infty}=m$ according to (10). Comparing (21) and (30), we arrive at the following dictionary

$$
O_{\eta}=2 \mathcal{N}\left(\frac{r_{0}^{2}}{2}\right)^{2} m^{2} f_{1}
$$

\subsection{Mass diffusion rate and susceptibility}

The mass operator $O_{\eta}$ can lead to fluctuation of axial charge. It is well known that the origin of axial charge fluctuation from QCD anomaly is topological transitions. The counterpart for $O_{\eta}$ is helicity flipping from elementary scattering [39, 40]. The rate of axial charge generation in case of topological transition is given by CS diffusion rate. Similarly, the corresponding rate in case of helicity flipping is given by the diffusion rate of $O_{\eta}$, which we calculate below. The diffusion rate of $O_{\eta}$ is encoded in the low frequency limit of retarded correlator. To calculate the retarded correlator, we need to turn on source for $\phi$ while keeping $A_{z}$ vanish on the boundary. Both $\phi$ and $A_{z}$ satisfy infalling wave condition on the horizon. It follows from (31) that the retarded correlator is given by

$$
G_{\eta \eta}(\omega)=\int d t\left\langle\left[O_{\eta}(t), O_{\eta}(0)\right]\right\rangle \Theta(t) e^{i \omega t}=-2 \mathcal{N}\left(\frac{r_{0}^{2}}{2}\right)^{2} m^{2} \frac{f_{1}}{f_{0}}
$$

The diffusion rate is defined by

$$
\Gamma_{m}=\lim _{\omega \rightarrow 0} \frac{2 i T}{\omega} G_{\eta \eta}(\omega)
$$

\footnotetext{
${ }^{2}$ Counter terms proportional to $B^{2}$ can in principle exist, but are not found in this case.
} 

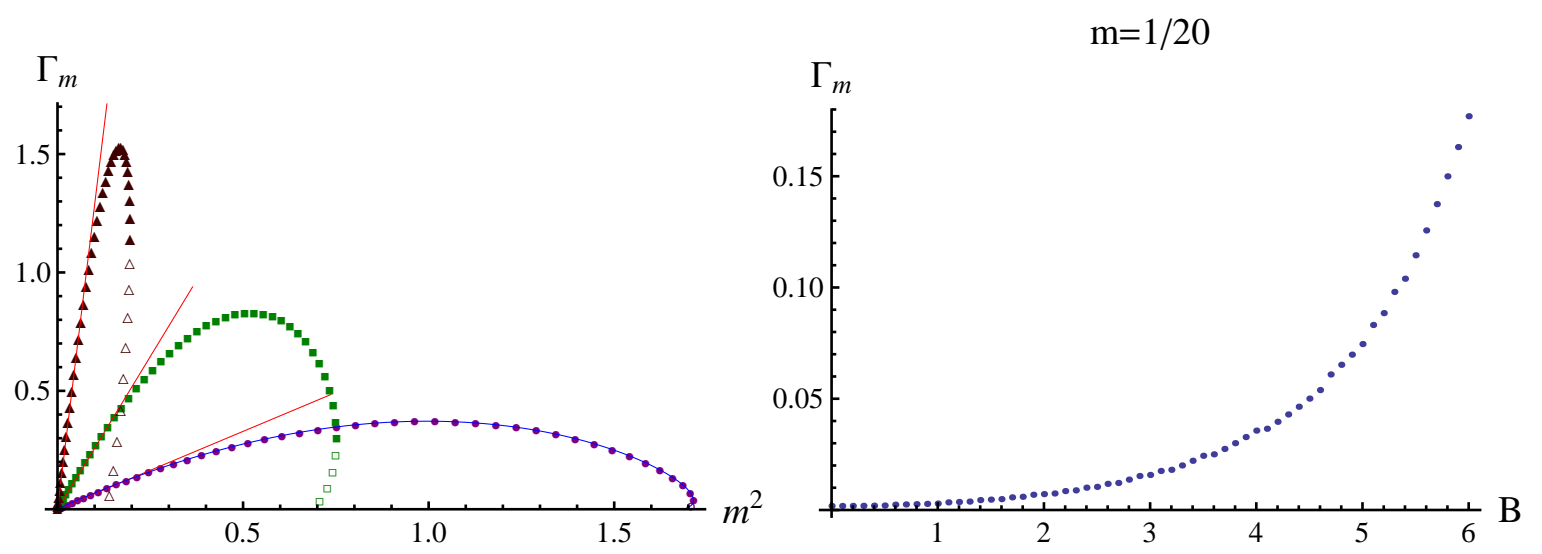

Figure 2: (left) The mass diffusion rate $\Gamma_{m}$ as a function of $m^{2}$ for $B=0$ (blue point), $B=2$ (purple square), $B=4$ (brown diamond). The units are set by $\pi T=1$. The blue line is given by (36) which fits well for $B=0$. To guide eyes, we also include linear fittings (red line) in the small mass region. The linear behavior is consistent with field theory expectation. We have used empty symbols for points in metastable phases. (right) $\Gamma_{m}$ as a function of $B$ at $m=1 / 20$. A rapid growth of $\Gamma_{m}$ with $B$ is found.

For the case $B=0$, there is no mixing between $\phi$ and $A_{z}$. We can simply use $\phi^{(0)}$ (54) in appendix B:

$$
\phi^{(0)}(\rho)=1-\frac{i \omega}{2 r_{0}}\left[\int_{1}^{\rho} d \rho^{\prime}\left(\frac{\left(\frac{r_{0}^{2}}{2}\right)^{2} 8 \cos ^{3} \theta_{h} \sin ^{2} \theta_{h}}{\sqrt{-h\left(\rho^{\prime}\right)} g^{\rho \rho}\left(\rho^{\prime}\right) g_{\phi \phi}\left(\rho^{\prime}\right)}-\frac{1}{\rho^{\prime}-1}\right)+\ln (\rho-1)\right] .
$$

This gives the following retarded correlator of $O_{\eta}$ :

$$
G_{\eta \eta}(\omega)=-2 \mathcal{N}\left(\frac{r_{0}^{2}}{2}\right)^{2} \frac{i \omega}{4 r_{0}} 8 \cos ^{3} \theta_{h} \sin ^{2} \theta_{h}
$$

(35) gives a mass diffusion rate $\Gamma_{m}$ as analog of CS diffusion rate:

$$
\Gamma_{m}=\frac{\mathcal{N}}{\pi}\left(\frac{r_{0}^{2}}{2}\right)^{2} 8 \cos ^{3} \theta_{h} \sin ^{2} \theta_{h}
$$

The dependence on $m$ is encoded in the combination of trigonometric functions, which clearly indicates an upper bound of the mass diffusion rate. We also extract $\Gamma_{m}$ using (33) with numerical solutions for general $B$ and $m$ in the meson melting phase. We plot numerical results of $\Gamma_{m}$ as a function of $m^{2}$ for different values of $B$ in Figure. 2. The case $B=0$ agrees well with analytic expression (35). We find the mass diffusion rate is a non-monotonous function of $m$. This is not difficult to understand: in the limit $m \rightarrow 0, \Gamma_{m}$ obviously should vanish as $O_{\eta} \sim m$. When $m$ approaches the phase boundary between meson melting 
phase and mesonic phase, we also expect helicity flipping to freeze due to formation of meson bound states. In between, there must be a maximum for $\Gamma_{m}$. Furthermore, the linear behavior of $\Gamma_{m}-m^{2}$ plot in small $m$ region supports the scaling $\Gamma_{m} \sim m^{2}$, which is consistent with field theory expectation. The $B$ dependence is more interesting: $\Gamma_{m}$ shows rapid growth with $B$. The presence of $B$ enhances the diffusion, which cannot be explained as the increase of effective mass. The enhancement of helicity flipping might provide a way to generate axial charge more efficiently. It is worth mentioning that an enhancement of CS diffusion rate due to magnetic field was also obtained in [41,42].

We would like to comment on the diffusive behavior of $O_{\eta}$. On general ground, the mass diffusion effect leads to accumulation of axial charge, which prevents its further generation. It would lead to modification of the long time (low frequency) behavior of $G_{\eta \eta}^{R}$. However, this does not happen due to the existence of the adjoint reservoir. The generated axial charge entirely dissipates to the adjoint reservoir. To see that, we compare $O_{\eta}$ and $O_{\text {loss }}$, which are the same quantity below evaluated at $\rho=\infty$ and $\rho=1$ respectively.

$$
\mathcal{N} \sqrt{-h} g^{\rho \rho} \rho_{\phi \phi} \phi^{\prime}
$$

It follows from the EOM (27) that the above quantity is constant in the limit $\omega \rightarrow 0$,

$$
\partial_{\rho}\left(\mathcal{N} \sqrt{-h} g^{\rho \rho} \rho_{\phi \phi} \phi^{\prime}\right)=0
$$

meaning that the generated charge is entirely balanced by the loss to the reservoir. Consequently, the low frequency behavior of $O_{\eta}$ correlator is still diffusive.

Turing on the source for $O_{\eta}$ also allows us to study the susceptibility of axial charge. In the presence of finite quark mass, the axial charge is not even approximately conserved, making the susceptibility a subtle concept. Following [31], we can use CME to define a dynamical susceptibility $\chi$. In the present model, it is given by

$$
\chi=\frac{n_{5}}{\mu_{5}}=\frac{\mathcal{N} B n_{5}}{J^{z}} .
$$

We need to calculate both $n_{5}$ and $J^{z}$ from response to source for $O_{\phi}$ in the hydrodynamic limit. $n_{5}$ is essentially known already. Denoting the source by $f_{m}$, we can express $n_{5}$ as

$$
-i \omega n_{5}(\omega)=O_{\eta}(\omega)=-G_{\eta \eta}^{R}(\omega) f_{m}(\omega) \sim O(\omega) f_{m}(\omega) .
$$

Therefore we obtain $n_{5} \sim O\left(\omega^{0}\right) f_{m}(\omega)$. On the other hand, $J^{z}$ is calculated using the dictionary (30). It is generated through the mixing between $\phi$ and $A_{z} . J^{z}$ is also expressible as response to $f_{m}$

$$
J^{z}(\omega)=-G_{j \eta}^{R}(\omega) f_{m}(\omega)
$$


Using the hydrodynamic solution (63) in appendix B and the dictionary (30), we find that there are two contributions to $G_{j \eta}^{R}$, both of which are of order $O(\omega B)$. Therefore we have $J^{z} \sim O(\omega B) f_{m}(\omega)$. Plugging the above qualitative results into (39), we obtain

$$
\chi \sim O\left(\omega^{-1}\right)
$$

It simply means that the susceptibility is divergent in the static limit $\omega \rightarrow 0$. Recalling that the susceptibility is well-defined in the massless limit, we arrive at the non-commutativity of the limits $m \rightarrow 0$ and $\omega \rightarrow 0$. The physical reason for divergent susceptibility is not difficult to understand. On on hand, the mass diffusion effect can spontaneously generate axial charge density at the cost of no energy. On the other hand, as we have seen already, the adjoint reservoir is a perfect sink for axial charge in the flavor sector, preventing accumulation of axial charge. Consequently, the axial charge can be continuously generated in the flavor sector. Note that the situation is different in case of axial charge generation by QCD anomaly. There the breaking of axial symmetry is suppressed by $1 / N_{c}$ (or the quenched limit), resulting in a finite dynamical susceptibility.

\subsection{Mass dissipation effect}

To study the mass dissipation effect, we turn on an electric field in $z$-direction by a time dependent $A_{z}$ on the boundary. We do not need to source $\phi$ on the boundary. Its profile is entirely generated via mixing of $A_{z}$ and $\phi$ in the bulk. The resulting $O_{\eta}$ from nontrivial profile of $\phi$ corresponds to the mass dissipation effect we are after. We also impose infalling wave boundary condition for $\phi$ and $A_{z}$ since we are interested in calculating response. We define the dimensionless mass dissipation rate

$$
r=\frac{O_{\eta}}{\mathcal{N} E \cdot B}
$$

The rate is a function of $\omega, m$ and $B$. In the hydrodynamic limit $\omega \rightarrow 0$, we can show that $r(\omega \rightarrow 0)$ is a real function of $m$ and $B$. In fact it can be related to embedding function for given $m$ and $B$ in the meson melting phase. To obtain $r(\omega \rightarrow 0)$ analytically, we need to solve the coupled EOM (27) in the hydrodynamic limit. The hydrodynamic solutions can be found in appendix B. We simply quote the results here. The leading nontrivial order is the zeroth order for $A_{z}$ and the first order for $\phi$ :

$$
\begin{aligned}
& A_{z}^{(0)}=a_{0}, \\
& \phi^{(1)}=\frac{\left(1-\cos ^{4} \theta_{h}\right) B i \omega a_{0}}{\left(\frac{r_{0}^{2}}{2}\right)^{2} m^{2}(-2)} \rho^{-2}+\cdots,
\end{aligned}
$$




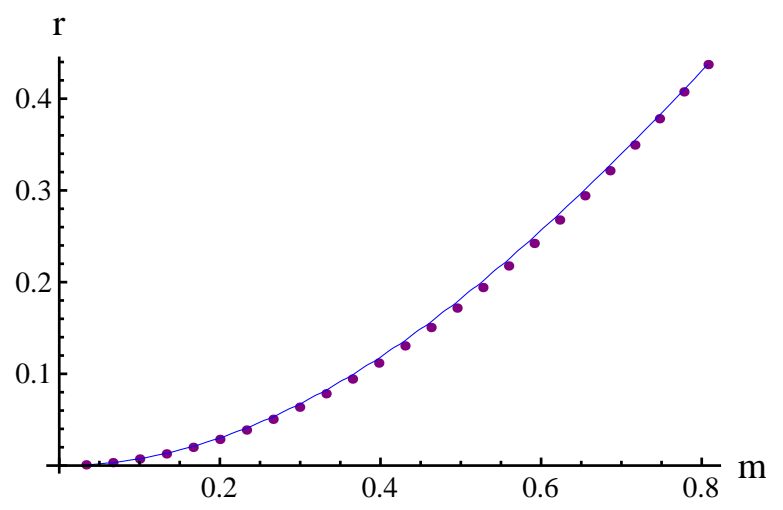

Figure 3: The mass dissipation rate $r$ as a function of $m$ from numerics with small $B$ and small $\omega$. It is a monotonous increasing function of $m$ as expected. The analytic function (45) is drawn in blue line and fits the numerical results well. The units are set by $\pi T=1$.

where $\theta_{h}$ is the value of $\theta$ on the horizon, which needs to be obtained from numerical embedding function for given $m$ and $B$. For $\phi^{(1)}$, we only retain its asymptotic behavior relevant for extracting $O_{\eta}$. (44) leads to the following rate

$$
r=1-\cos ^{4} \theta_{h}
$$

We also study the rate of dissipation by numerical solutions. In practice, we generate two independent infalling numerical solutions at the horizon and use their linear combination to construct the solution with desired boundary condition. We show $m$-dependence of $r$ in the limit $B=0$ and $B$-dependence of $r$ at different values of $m$ in Figure 3 and Figure 4 . We find good agreement with analytic expression (45). On general ground, we expect the rate to be a monotonous increasing function of $m$. In particular, $r \rightarrow 0$ as $m \rightarrow 0$. Indeed, this is confirmed in Fig. 3. We further note that the effect of $B$ enhances the dissipation on top of the mass effect in Fig. 4. The physical interpretation of the dissipation rate $r$ turns out to be a subtle question. Recalling the axial anomaly equation (22), we would draw the following conclusion: for every one unit of axial charge generated by parallel electric and magnetic field, $r$ unit of it dissipates through the mass term, with a unit of $1-r$ axial charge remaining. The remaining axial charge survives even in the hydrodynamic limit since we have $\omega \rightarrow 0$. This is not true because we have ignored a third source of axial charge dissipation, i.e. loss to the adjoint reservoir. The anomaly equation (22) should be supplemented by the loss rate

$$
\partial_{\mu} J_{R}^{\mu}=O_{\eta}+\mathcal{N} E \cdot B-O_{\mathrm{loss}},
$$




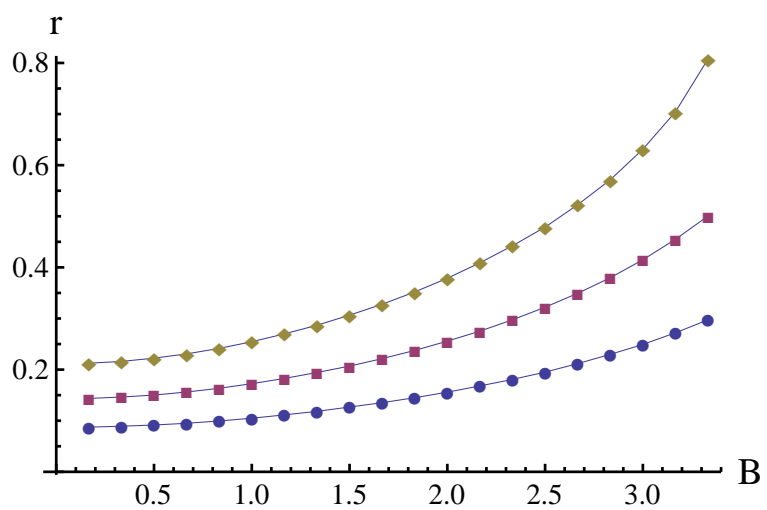

Figure 4: The mass dissipation rate $r$ as a function of $B$ for $m=7 / 20$ (blue point), $m=9 / 20$ (purple square), and $m=11 / 20$ (brown diamond). The units are set by $\pi T=1$. The analytic function (45) is drawn in blue line and fits the numerical results well. It is a monotonous increasing function of $B$.

with the explicit form of loss rate given by

$$
O_{\text {loss }}=\left.\mathcal{N} \sqrt{-h} g^{\rho \rho} r_{\phi \phi} \partial_{\rho} \phi\right|_{\rho \rightarrow 1}+\left.\mathcal{N} \Omega E \cdot B\right|_{\rho \rightarrow 1}
$$

It is known that the loss rate can be IR unsafe [43]. Indeed, plugging in the hydrodynamic solution $A_{z}^{(0)}$ and $\phi^{(1)}$ in appendix B into (46), we find both terms becomes infinitely oscillatory as $\rho \rightarrow 1$. Nevertheless we can still extract useful information by taking the hydrodynamic limit $\omega \rightarrow 0$ before the IR limit $\rho \rightarrow 1$. Using this regularization we find the $\mathcal{N} \Omega E \cdot B$ term becomes $\mathcal{N} \cos ^{4} \theta_{h} E \cdot B$, while the other term is higher order in $\omega$. We immediately note that $\mathcal{N} \cos ^{4} \theta_{h} E \cdot B$ is precisely the $1-r$ unit of axial charge. Subtracting the charge loss to the reservoir, we find only $r$ unit of axial charge is effectively generated in the flavor sector by parallel electric and magnetic field. All dissipates by the mass term. This simply means no axial charge survives in the hydrodynamic limit. After clarifying the role of axial charge loss to adjoint reservoir, we should interpret $r$ as a measure of mass dissipation effect compared to dissipation to the adjoint reservoir. The dissipation through mass term is favored at large $m$ and $B$.

The statement on the non-survival of axial charge can receive correction higher order in $\omega$, which quantifies the charge survival rate. We can compare with the relaxation time approximation employed in [32,33], in which the following form of axial anomaly equation is assumed (here we use $r \mathcal{N} E \cdot B$ for effectively axial charge generation)

$$
\partial_{t} n_{5}=-\frac{n_{5}}{\tau}+r \mathcal{N} E \cdot B,
$$


with $\tau$ being the relaxation time. Physically it means the presence of axial charge $n_{5}$ induces $O_{\eta}=-\frac{n_{5}}{\tau}$. Plugging it into (48), we can solve for $O_{\eta}$ in frequency space

$$
O_{\eta}=-\frac{r \mathcal{N} E \cdot B}{1-i \omega \tau}
$$

The leading order result $O_{\eta}=-r \mathcal{N} E \cdot B$ corresponds to our result of full dissipation. In principle, by going to high order in $\omega$, we could calculate the relaxation time $\tau$. We will not attempt it in this paper.

\section{Summary}

We have investigated the effect of finite quark mass and magnetic field in the generation and dissipation of axial charge, using a D3/D7 model. For axial charge generation, we calculated the mass diffusion rate. It is analogous to the Chern-Simon diffusion rate as a measure of axial charge fluctuation. The mass diffusion rate is a bounded non-monotonous function of mass at vanishing magnetic field. The presence of magnetic field enhances the diffusion. At small $m$, our numerical results are consistent with an approximate scaling for the mass diffusion rate

$$
\Gamma_{m} \sim m^{2} F(B)
$$

with $F(B)$ a rapid growing function in the meson melting phase. We also defined a dynamical susceptibility of axial charge using CME. We found the susceptibility to be divergent in the static limit $\omega \rightarrow 0$. It is due to two reasons: $i$, spontaneous generation of axial charge by mass diffusion effect; ii, continuous leakage of axial charge from flavor sector to the adjoint sector, preventing the accumulation of axial charge.

For axial charge dissipation, we found that a mass term is induced in the presence of parallel electric and magnetic fields, reducing the generation of axial charge. After carefully subtracting the axial charge loss rate to the adjoint sector, we found that the axial charge dissipate entirely through the mass term in the long time limit. To the order we consider, it is consistent with a relaxation time approximation.

\section{Acknowledgments}

We thank K. Landsteiner and Y. Yin for critical comments on an early version of the paper. We also thank D. Kharzeev, J. F. Liao, Y. Liu, L. Yaffe, H.-U. Yee and Y. Yin for useful discussions. The work of S.L. is in part supported by Junior Faculty's Fund at Sun Yat-Sen University. 


\section{A Phase diagram at finite $m$ and $B$}

It is known that D3/D7 brane system as a model for finite temperature QGP in the quenched limit has a first order phase transition [35 38]. At large quark mass and strong magnetic field (with temperature fixed), the probe D7 brane lie outside of the black hole horizon. In this phase the meson stays in bound state and its spectrum possesses a mass gap. At small quark mass and weak magnetic field, the brane crosses the horizon and this corresponds to the meson melting phase. The case in which the D7 branes touch the horizon corresponds to critical embedding, giving rise to critical mass and condensate. The embeddings close to the critical embedding show oscillatory behavior for the corresponding mass and condensate parameters around their critical values. This implies that the condensate is a multivalued function of mass, corresponding to different states. The true ground state is determined by the embedding that minimizes the free energy. Denoting $\chi=\sin \theta$, we can rewrite the action (12) as

$$
S_{D B I}=-\mathcal{N} \int d \rho \frac{\left(1-\rho^{4}\right)\left(1-\chi^{2}\right) \sqrt{\left(1-\chi^{2}+\rho^{2} \chi^{\prime 2}\right)\left(1+\left(2+4 B^{2}\right) \rho^{4}+\rho^{8}\right)}}{4 \rho^{5}},
$$

where we have set $r_{0}=1$, which amounts to fixing the temperature $T=\frac{1}{\pi}$. The EOM following from (51) is solved by numerical integration of the EOM. The black hole embedding and Minkowski embedding satisfy different boundary conditions. For black hole embedding, the boundary condition is $\chi(\rho=1)=\chi_{0}, \chi^{\prime}(\rho=1)=0$, with integration domain from $\rho=1$ to $\rho=\rho_{\max }$. For Minkowski embedding, the boundary condition is $\chi\left(\rho=\rho_{\min }\right)=1$, $\chi^{\prime}\left(\rho=\rho_{\min }\right)=\frac{\left(1-\rho^{4}\right)\left(1+\left(2+4 B^{2}\right) \rho^{4}+\rho^{8}\right)}{\rho\left(1+\rho^{4}\right)\left(1+2 B^{2} \rho^{4}+\rho^{8}\right)}$, with integration domain from $\rho=\rho_{\min }>1$ to $\rho=\rho_{\max }$. The initial condition for the derivative is chosen such that $\chi^{\prime \prime}\left(\rho=\rho_{\min }\right)$ can be uniquely determined by EOM. In practice, we start the integration at $\rho=1+\epsilon$ for black hole embedding and $\rho=\rho_{\min }+\epsilon$ for Minkowski embedding.

We note that the free energy $F=T S$ contains a UV divergence and therefore needs to be renormalized. Following [35], we add to the action the counter term

$$
S_{\text {counter }}=-\frac{\mathcal{N}}{4}\left[\left(\left(\rho_{\max }^{2}-m^{2}\right)^{2}-4 m c\right)+\frac{B^{2}}{2} \ln \rho_{\max }\right]
$$

Note the appearance of a new term due to magnetic field as compared to [35]. The renormalized action $S=S_{D B I}+S_{\text {counter }}$ is finite as we take $\rho_{\max } \rightarrow \infty$. The true ground state is found by comparing the free energy of black hole embedding and Minkowski embedding, corresponding to meson melting phase and mesonic phase. The phase transition is first order and present below a certain critical magnetic field $B_{c}$. Above $B_{c}$, only Minkowski 

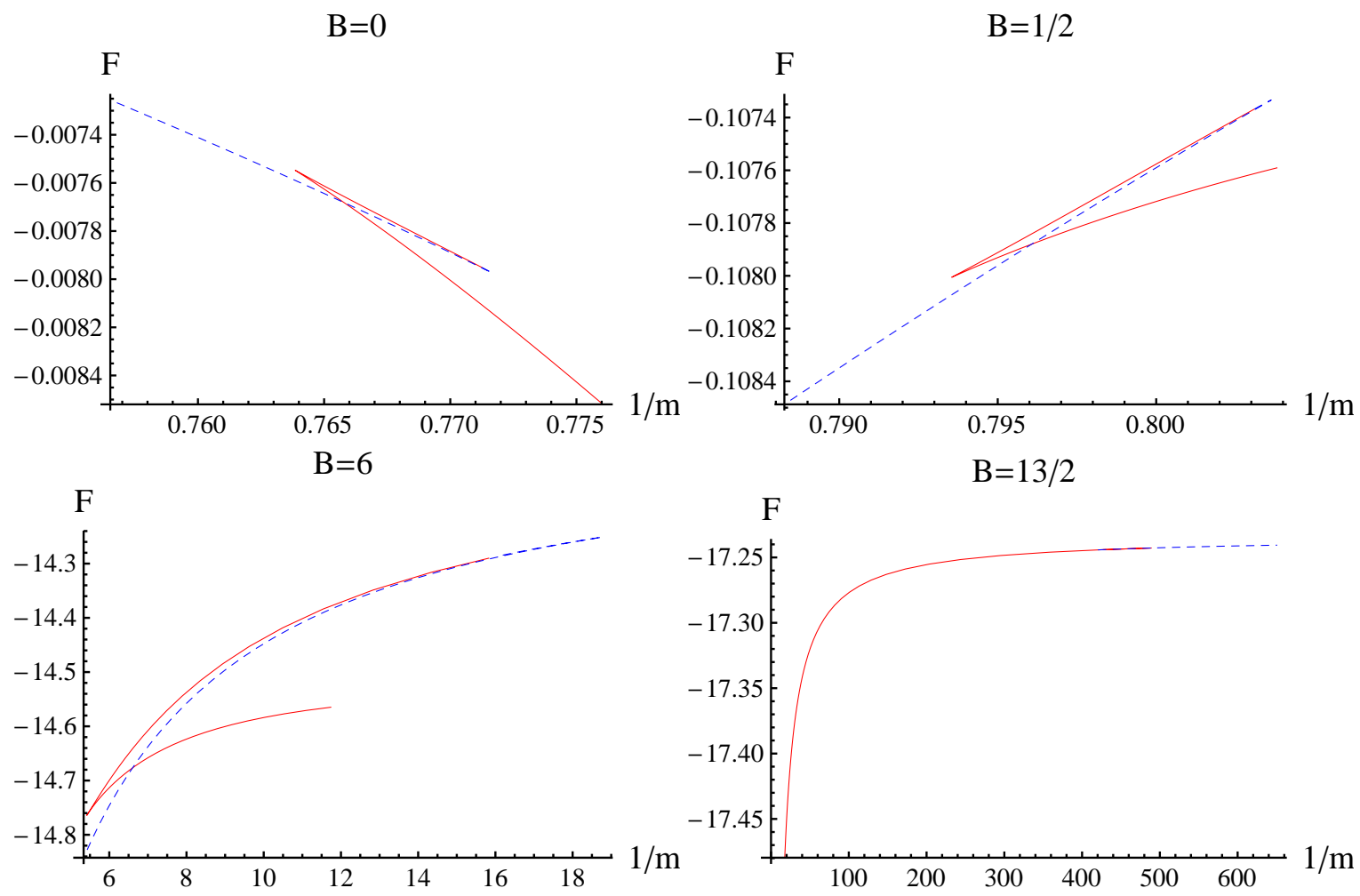

Figure 5: Free energy $F$ as a function of $1 / m$ at different $B$ for $D 3 / D 7$ system. The units are set by $\pi T=1$. The red continuous (blue dashed) curves correspond to the black hole (Minkowski) embedding.

embedding is possible. Below $B_{c}$, metastable phases of black hole embedding are found as we increases $B$. We illustrate the structure of metastable phases in Figure 5 .

\section{B Hydrodynamic solution of fluctuations}

We wish to solve (27) in the hydrodynamic limit. We reproduce (27) for convenience.

$$
\begin{aligned}
& \omega^{2} \sqrt{-h} g^{t t} g_{\phi \phi} \phi-\partial_{\rho}\left(\sqrt{-h} g^{\rho \rho} g_{\phi \phi} \phi^{\prime}\right)-B \partial_{\rho}\left(\cos ^{4} \theta\right) A_{z}(-i \omega)=0, \\
& \omega^{2} \sqrt{-h} g^{t t} g^{x x} A_{z}-\partial_{\rho}\left(\sqrt{-h} g^{\rho \rho} g^{x x} A_{z}^{\prime}\right)+B \partial_{\rho}\left(\cos ^{4} \theta\right) \phi(-i \omega)=0 .
\end{aligned}
$$

For pedagogical reason, we work in the small $B$ limit and solve (53) order by order in $B$. Since correction to $\sqrt{-h}$ starts from $O\left(B^{2}\right)$, we can simply use the $B=0$ limit of $\sqrt{-h}$ for the solution up to $O(B)$. The order $O\left(B^{0}\right)$ solution satisfies homogeneous equation. In the 
hydrodynamic regime, the solution is given by

$$
\begin{aligned}
& \phi^{(0)}(\rho)=1-\frac{i \omega}{2 r_{0}}\left[\int_{1}^{\rho} d \rho^{\prime}\left(\frac{\left(\frac{r_{0}^{2}}{2}\right)^{2} 8 \cos ^{3} \theta_{h} \sin ^{2} \theta_{h}}{\sqrt{-h\left(\rho^{\prime}\right)} g^{\rho \rho}\left(\rho^{\prime}\right) g_{\phi \phi}\left(\rho^{\prime}\right)}-\frac{1}{\rho^{\prime}-1}\right)+\ln (\rho-1)\right], \\
& A_{z}^{(0)}(\rho)=1-\frac{i \omega}{2 r_{0}}\left[\int_{1}^{\rho} d \rho^{\prime}\left(\frac{\frac{r_{0}^{2}}{2} 4 \cos ^{3} \theta_{h}}{\sqrt{-h\left(\rho^{\prime}\right)} g^{\rho \rho}\left(\rho^{\prime}\right) g^{x x}\left(\rho^{\prime}\right)}-\frac{1}{\rho^{\prime}-1}\right)+\ln (\rho-1)\right] .
\end{aligned}
$$

We have chosen a specific normalization for the homogeneous solutions. At order $O(B)$, we need to solve the inhomogeneous equations sourced by the mixing terms. This can be achieved by using Green's function for $\phi$ and $A_{z}$, which are defined by

$$
\begin{aligned}
& \partial_{\rho}^{2} \mathcal{G}_{\phi}\left(\rho, \rho^{\prime}\right)+\partial_{\rho} \mathcal{G}_{\phi}\left(\rho, \rho^{\prime}\right) \partial_{\rho} \ln \left(\sqrt{-h} g^{\rho \rho} g_{\phi \phi}\right)-\frac{\omega^{2} g^{t t}}{g^{\rho \rho}} \mathcal{G}_{\phi}\left(\rho, \rho^{\prime}\right)=\delta\left(\rho-\rho^{\prime}\right) \\
& \partial_{\rho}^{2} \mathcal{G}_{A}\left(\rho, \rho^{\prime}\right)+\partial_{\rho} \mathcal{G}_{A}\left(\rho, \rho^{\prime}\right) \partial_{\rho} \ln \left(\sqrt{-h} g^{\rho \rho} g^{x x}\right)-\frac{\omega^{2} g^{t t}}{g^{\rho \rho}} \mathcal{G}_{A}\left(\rho, \rho^{\prime}\right)=\delta\left(\rho-\rho^{\prime}\right)
\end{aligned}
$$

We require that the inhomogeneous solutions satisfy the infalling wave condition on the horizon and vanish on the boundary. It is convenient to construct the Green's function using two independent solutions satisfying the above boundary conditions. We illustrate the procedure using $\mathcal{G}_{\phi}$ as an example. The two independent solutions are chosen as below:

$$
\begin{aligned}
& \phi_{h}=(\rho-1)^{-\frac{i \omega}{2 r_{0}}}(1+\cdots)=h_{0}+\frac{h_{1}}{\rho^{2}}+\cdots, \\
& \phi_{b}=\phi_{h} h_{0}^{*}-\phi_{h}^{*} h_{0}=\left(h_{0}^{*} h_{1}-h_{0} h_{1}^{*}\right) \rho^{-2}+\cdots .
\end{aligned}
$$

Here $\phi_{h}$ satisfies the infalling wave condition on the horizon. $h_{0}$ and $h_{1}$ (not to be confused with $h$ ) are coefficients of asymptotic expansion of $\phi_{h}$. $\phi_{b}$ is constructed from linear combination of $\phi_{h}$ and its complex conjugate such that it vanishes on the boundary. $\mathcal{G}_{\phi}$ can be constructed as follows

$$
\mathcal{G}_{\phi}\left(\rho, \rho^{\prime}\right)=\frac{1}{\phi_{b}^{\prime}\left(\rho^{\prime}\right) \phi_{h}\left(\rho^{\prime}\right) \phi_{b}\left(\rho^{\prime}\right) \phi_{h}^{\prime}\left(\rho^{\prime}\right)}\left[\phi_{h}\left(\rho^{\prime}\right) \phi_{b}(\rho) \theta\left(\rho-\rho^{\prime}\right)+\phi_{b}\left(\rho^{\prime}\right) \phi_{h}(\rho) \theta\left(\rho^{\prime}-\rho\right)\right] .
$$

The Wronskian appearing in (57) can be fixed up to normalization from the homogeneous equation:

$$
\phi_{b}^{\prime} \phi_{h}-\phi_{h}^{\prime} \phi_{b}=\frac{\#}{\sqrt{-h} g^{\rho \rho} g_{\phi \phi}} .
$$

We can fixed the normalization by taking the limit $\rho \rightarrow \infty$ of (58). Comparing the limit with (156), we obtain

$$
\#=\left(\frac{r_{0}^{2}}{2}\right)^{2} m^{2}\left(h_{0}^{*} h_{1}-h_{0} h_{1}^{*}\right)(-2) h_{0}
$$


where we used the fact $\left.\rho \sin \theta\right|_{\rho \rightarrow \infty}=m$. The inhomogeneous solution is given by the convolution of Green's function and corresponding source

$$
\phi^{(1)}(\rho)=\int_{1}^{\infty} d \rho^{\prime} \mathcal{G}_{\phi}\left(\rho, \rho^{\prime}\right) s\left(\rho^{\prime}\right)
$$

with the source

$$
s(\rho)=\frac{B \partial_{\rho}\left(\cos ^{4} \theta\right) A_{z}^{(0)} i \omega}{\sqrt{-h} g^{\rho \rho} g_{\phi \phi}} .
$$

We are only interested in the limit $\rho \rightarrow \infty$ of (60), which is

$$
\phi^{(1)}(\rho)=\int_{1}^{\infty} d \rho^{\prime} \frac{\phi_{h}\left(\rho^{\prime}\right) B \partial_{\rho^{\prime}}\left(\cos ^{4} \theta\left(\rho^{\prime}\right)\right) A_{z}^{(0)}\left(\rho^{\prime}\right) i \omega}{\left(\frac{r_{0}^{2}}{2}\right)^{2} m^{2}(-2) h_{0}} \rho^{-2}+\cdots .
$$

Following the same procedure, we obtain the counterpart of $A_{z}$ :

$$
A_{z}^{(1)}(\rho)=\int_{1}^{\infty} d \rho^{\prime} \frac{-A_{z, h}\left(\rho^{\prime}\right) B \partial_{\rho^{\prime}}\left(\cos ^{4} \theta\left(\rho^{\prime}\right)\right) \phi^{(0)}\left(\rho^{\prime}\right) i \omega}{\left(\frac{r_{0}^{2}}{2}(-2) a_{0}\right)} \rho^{-2}+\cdots,
$$

where $A_{z, h}$ is defined as the solution satisfying infalling wave condition on the horizon, with boundary value $a_{0}$. Before closing this section, we claim that (54), (62) and (63) can also be understood as series expansions in $\omega$ : we should discard the $O(\omega)$ terms in (54) and view the rest as zeroth solution. The first order solutions get contribution from the discarded terms in (54), (62) and (63). Furthermore, we can allow for arbitrary dependence of $\sqrt{-h}$ on $B$ in (54), (62) and (63) provided that we work with sufficient small $\omega$.

\section{References}

[1] Dmitri E. Kharzeev, Larry D. McLerran, and Harmen J. Warringa. The Effects of topological charge change in heavy ion collisions: 'Event by event $\mathrm{P}$ and $\mathrm{CP}$ violation'. Nucl.Phys., A803:227-253, 2008.

[2] D. Kharzeev and A. Zhitnitsky. Charge separation induced by P-odd bubbles in QCD matter. Nucl.Phys., A797:67-79, 2007.

[3] Dmitri Kharzeev. Parity violation in hot QCD: Why it can happen, and how to look for it. Phys.Lett., B633:260-264, 2006.

[4] Kenji Fukushima, Dmitri E. Kharzeev, and Harmen J. Warringa. The chiral magnetic effect. Phys. Rev. D, 78:074033, 2008. 
[5] Dmitri E. Kharzeev and Ho-Ung Yee. Chiral Magnetic Wave. Phys.Rev., D83:085007, 2011.

[6] Yannis Burnier, Dmitri E. Kharzeev, Jinfeng Liao, and Ho-Ung Yee. Chiral magnetic wave at finite baryon density and the electric quadrupole moment of quark-gluon plasma in heavy ion collisions. Phys. Rev. Lett., 107:052303, 2011.

[7] D. E. Kharzeev, J. Liao, S. A. Voloshin, and G. Wang. Chiral magnetic and vortical effects in high-energy nuclear collisions-A status report. Prog. Part. Nucl. Phys., $88: 1-28,2016$.

[8] Jinfeng Liao. Anomalous transport effects and possible environmental symmetry 'violation' in heavy-ion collisions. Pramana, 84(5):901-926, 2015.

[9] Xu-Guang Huang. Electromagnetic fields and anomalous transports in heavy-ion collisions - A pedagogical review. 2015.

[10] Dam T. Son and Piotr Surowka. Hydrodynamics with triangle anomalies. Phys. Rev. Lett., 103:191601, 2009.

[11] Yin Jiang, Xu-Guang Huang, and Jinfeng Liao. Chiral vortical wave and induced flavor charge transport in a rotating quark-gluon plasma. Phys. Rev., D92(7):071501, 2015.

[12] L. Adamczyk et al. Beam-energy dependence of charge separation along the magnetic field in $\mathrm{Au}+\mathrm{Au}$ collisions at RHIC. Phys.Rev.Lett., 113:052302, 2014.

[13] B.I. Abelev et al. Azimuthal Charged-Particle Correlations and Possible Local Strong Parity Violation. Phys.Rev.Lett., 103:251601, 2009.

[14] Betty Abelev et al. Charge separation relative to the reaction plane in $\mathrm{Pb}-\mathrm{Pb}$ collisions at $\sqrt{s_{N N}}=2.76 \mathrm{TeV}$. Phys.Rev.Lett., 110(1):012301, 2013.

[15] Dmitri E. Kharzeev and Ho-Ung Yee. Anomalies and time reversal invariance in relativistic hydrodynamics: the second order and higher dimensional formulations. Phys.Rev., D84:045025, 2011.

[16] Eugenio Megias and Francisco Pena-Benitez. Holographic Gravitational Anomaly in First and Second Order Hydrodynamics. JHEP, 1305:115, 2013.

[17] Kristan Jensen, Pavel Kovtun, and Adam Ritz. Chiral conductivities and effective field theory. JHEP, 1310:186, 2013. 
[18] A. V. Sadofyev and M. V. Isachenkov. The Chiral magnetic effect in hydrodynamical approach. Phys. Lett., B697:404-406, 2011.

[19] Shi Pu, Jian-hua Gao, and Qun Wang. A consistent description of kinetic equation with triangle anomaly. Phys.Rev., D83:094017, 2011.

[20] Jiunn-Wei Chen, Shi Pu, Qun Wang, and Xin-Nian Wang. Berry Curvature and FourDimensional Monopoles in the Relativistic Chiral Kinetic Equation. Phys.Rev.Lett., 110(26):262301, 2013.

[21] M.A. Stephanov and Y. Yin. Chiral Kinetic Theory. Phys.Rev.Lett., 109:162001, 2012.

[22] Mikhail Stephanov, Ho-Ung Yee, and Yi Yin. Collective modes of chiral kinetic theory in a magnetic field. Phys. Rev., D91(12):125014, 2015.

[23] Dam Thanh Son and Naoki Yamamoto. Berry Curvature, Triangle Anomalies, and the Chiral Magnetic Effect in Fermi Liquids. Phys.Rev.Lett., 109:181602, 2012.

[24] Dam Thanh Son and Naoki Yamamoto. Kinetic theory with Berry curvature from quantum field theories. Phys.Rev., D87(8):085016, 2013.

[25] Yan Wu, Defu Hou, and Hai-cang Ren. The Subtleties of the Wigner Function Formulation of the Chiral Magnetic Effect. 2016.

[26] Jiunn-Wei Chen, Jin-yi Pang, Shi Pu, and Qun Wang. Kinetic equations for massive Dirac fermions in electromagnetic field with non-Abelian Berry phase. Phys. Rev., D89(9):094003, 2014.

[27] V. P. Kirilin, A. V. Sadofyev, and V. I. Zakharov. Anomaly and long-range forces. In Proceedings, 100th anniversary of the birth of I.Ya. Pomeranchuk, pages 272-286, 2014 .

[28] Kenji Fukushima, Dmitri E. Kharzeev, and Harmen J. Warringa. Real-time dynamics of the Chiral Magnetic Effect. Phys.Rev.Lett., 104:212001, 2010.

[29] Amadeo Jimenez-Alba, Karl Landsteiner, and Luis Melgar. Anomalous magnetoresponse and the Stückelberg axion in holography. Phys.Rev., D90(12):126004, 2014.

[30] Ioannis Iatrakis, Shu Lin, and Yi Yin. Axial current generation by P-odd domains in QCD matter. Phys. Rev. Lett., 114(25):252301, 2015. 
[31] Ioannis Iatrakis, Shu Lin, and Yi Yin. The anomalous transport of axial charge: topological vs non-topological fluctuations. JHEP, 09:030, 2015.

[32] Amadeo Jimenez-Alba, Karl Landsteiner, Yan Liu, and Ya-Wen Sun. Anomalous magnetoconductivity and relaxation times in holography. JHEP, 07:117, 2015.

[33] Ya-Wen Sun and Qing Yang. Negative Magnetoresistivity in Holography. 2016.

[34] Carlos Hoyos, Tatsuma Nishioka, and Andy O'Bannon. A Chiral Magnetic Effect from AdS/CFT with Flavor. JHEP, 1110:084, 2011.

[35] David Mateos, Robert C. Myers, and Rowan M. Thomson. Holographic phase transitions with fundamental matter. Phys. Rev. Lett., 97:091601, 2006.

[36] Carlos Hoyos-Badajoz, Karl Landsteiner, and Sergio Montero. Holographic meson melting. JHEP, 04:031, 2007.

[37] Veselin G. Filev, Clifford V. Johnson, R. C. Rashkov, and K. S. Viswanathan. Flavoured large N gauge theory in an external magnetic field. JHEP, 10:019, 2007.

[38] Johanna Erdmenger, Rene Meyer, and Jonathan P. Shock. AdS/CFT with flavour in electric and magnetic Kalb-Ramond fields. JHEP, 12:091, 2007.

[39] Cristina Manuel and Juan M. Torres-Rincon. Dynamical evolution of the chiral magnetic effect: Applications to the quark-gluon plasma. Phys. Rev., D92(7):074018, 2015.

[40] Dorota Grabowska, David B. Kaplan, and Sanjay Reddy. Role of the electron mass in damping chiral plasma instability in Supernovae and neutron stars. Phys. Rev., D91(8):085035, 2015.

[41] Gokce Basar and Dmitri E. Kharzeev. The Chern-Simons diffusion rate in strongly coupled N=4 SYM plasma in an external magnetic field. Phys.Rev., D85:086012, 2012.

[42] Tara Drwenski, Umut Gursoy, and Ioannis Iatrakis. Thermodynamics and CP-odd transport in Holographic QCD with Finite Magnetic Field. 2015.

[43] Andreas Karch, Andy O'Bannon, and Ethan Thompson. The Stress-Energy Tensor of Flavor Fields from AdS/CFT. JHEP, 04:021, 2009. 